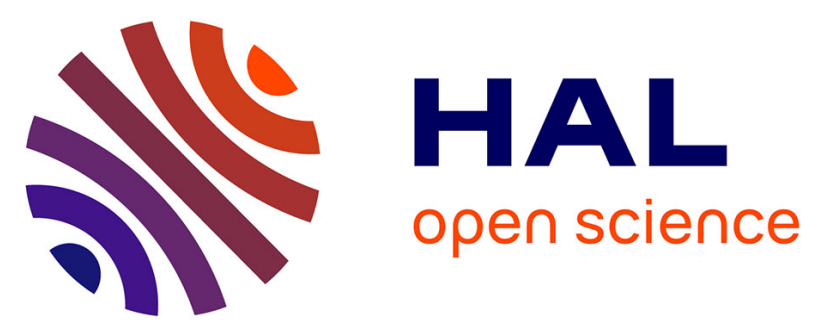

\title{
Multiaxial fatigue models for short glass fiber reinforced polyamide - Part I: Nonlinear anisotropic constitutive behavior for cyclic response
}

\author{
Antoine Launay, Habibou Maitournam, Yann Marco, Ida Raoult
}

\section{- To cite this version:}

Antoine Launay, Habibou Maitournam, Yann Marco, Ida Raoult. Multiaxial fatigue models for short glass fiber reinforced polyamide - Part I: Nonlinear anisotropic constitutive behavior for cyclic response. International Journal of Fatigue, 2013, 47, pp $382-389$, issn = "0142-1123", url $=$ "http://www.sciencedirect.com/science/article/pii/S014211231. 10.1016/j.ijfatigue.2012.03.012 . hal-00778277

\section{HAL Id: hal-00778277}

https://hal-polytechnique.archives-ouvertes.fr/hal-00778277

Submitted on 19 Jan 2013

HAL is a multi-disciplinary open access archive for the deposit and dissemination of scientific research documents, whether they are published or not. The documents may come from teaching and research institutions in France or abroad, or from public or private research centers.
L'archive ouverte pluridisciplinaire HAL, est destinée au dépôt et à la diffusion de documents scientifiques de niveau recherche, publiés ou non, émanant des établissements d'enseignement et de recherche français ou étrangers, des laboratoires publics ou privés. 


\title{
Multiaxial fatigue models for short glass fibre reinforced polyamide. Part I: Nonlinear anisotropic constitutive behaviour for the cyclic response.
}

\author{
A. Launay ${ }^{\mathrm{a}, \mathrm{b}, \mathrm{c}, *}$, M. H. Maitournam ${ }^{\mathrm{a}}$, Y. Marco ${ }^{\mathrm{b}}$, I. Raoult ${ }^{\mathrm{c}}$ \\ ${ }^{a}$ Laboratoire de Mécanique des Solides (CNRS UMR 7649), École polytechnique, 91128 Palaiseau, France \\ ${ }^{b}$ Laboratoire Brestois de Mécanique et des Systèmes (EA 4325 ENSTA Bretagne/UBO/ENIB), 2 rue François Verny, 29806 Brest Cedex 9 , France \\ ${ }^{c}$ PSA Peugeot Citroën (Direction Scientifique et des Technologies Futures), Route de Gisy, 78943 Vélizy-Villacoublay Cedex, France
}

\begin{abstract}
Components made of short fibre reinforced thermoplastics are increasingly used in the automotive industry, and more and more frequently subjected to fatigue loadings during their service life. The determination of a predictive fatigue criterion is therefore a serious issue for the designers, and requires the knowledge of the local mechanical response. As the cyclic behaviour of polymeric material is reckoned to be highly nonlinear, even at room temperature, an accurate constitutive model is a preliminary step for confident fatigue design.

This paper presents an extension of the constitutive behaviour proposed by the authors in a precedent work, in order to take into account the influence of the local fibre orientation distribution (FOD) on overall anisotropic elastic and viscoplastic properties. The proposed model is written in a general 3D anisotropic framework, and is validated on tensile samples with various FOD and loading histories: monotonic tensions, creep and/or relaxation steps, cyclic loadings.
\end{abstract}

Keywords: PA66 GF35, constitutive model, fibre orientation distribution, nonlinear behaviour, anisotropic

behaviour

\section{Introduction}

\subsection{Motivations}

In order to reduce their environmental impact, carmakers wish to substitute heavy metallic parts by lightweight composites structures. Short glass fibre reinforced (SGFR) thermoplastics are a cost-efficient solution which combines sufficient stiffness for many structural components and a large freedom of shapes provided by injection moulding. The choices of glass fibres and polyamide matrices are usual and motivated by cost and thermal stability respectively.

These materials have long been used by the automotive industry for components which do not undergo much mechanical loading. They are now increasingly used to make structural parts such as intake manifolds or engine mounts, which are subjected to complex and repeated mechanical loading as well as environmental conditions such as

\footnotetext{
${ }^{*}$ Corresponding author. Tel.: (+33/0)1 57594166 ; fax: (+33/0)1 41243256

Email address: antoine. launay@mpsa.com (A. Launay)
} 
heat and humidity. Therefore, the need for a method to design those components against fatigue failure has become a serious issue during the last years, when different authors began adressing the problem. For example, in part II of this work [1], De Monte's and Klimkeit's recent fatigue experiments give the basis for a comparative study of different fatigue criteria $[2,3]$.

\subsection{Approaches for fatigue design}

Fatigue damage, particularly for composite materials, is often considered as a continuous phenomena taking place all along the lifetime of the structure and sometimes modelled as such. Continuum damage mechanics is an approach which describes damage as an internal variable in the behaviour with its specific evolution law. Some authors have developped constitutive equations for reinforced thermoplastics [4,5]. The damage is zero for the virgin material and increases until it reaches the value of one which defines failure. Although this approach relies on physical basis, it is not convenient in an industrial context since it implies the simulation of every load cycle from the very beginning until the very end, when a macroscopic crack is initiated. The duration of such computations for automotive components is prohibitive.

In cases where the cyclic response of the structure reaches a steady state, an alternative approach consists of uncoupling the damage evolution from the constitutive behaviour. The local response of the material is then considered to be repeatable after a few cycles, and any relevant mechanical variable can be computed for the steady-state cycle, once and for all, and related to a number of cycles to failure. This approach has been successfully put into application in the automotive industry for fatigue design of metallic structures [6,7] as well as elastomeric components [8] undergoing nonlinear strain mechanisms. Experimental data from the literature regarding fatigue of SGFR thermoplastics [9-11] show that macroscopic quantities (e.g. secant stiffness, hysteresis, cyclic damping, cumulated remanent strain per cycle...) do not vary much during load-controlled fatigue tests, provided that heat build-up is negligible. This is the reason why we here adopt the second approach.

\subsection{Cyclic behaviour: scale transition method or macroscopic modelling?}

Because of their multiphasic nature, SGFR thermoplastics have been extensively modelled by scale transition methods. The macroscopic mechanical behaviour of the composite is deduced - through nonlinear homogenization schemes - from constitutive models of the fibres and the matrix and from a representation of the microstructure [1215]. The fibres are usually considered as elastic whereas the matrix is nonlinear, with varying degrees of complexity. These techniques yield interesting results since they naturally integrate the anisotropy in every aspects of the behaviour (including inelastic ones). Moreover, they provide statistical information on the mechanical state inside the different phases. However, they suffer one major drawback: because the process of homogenization must be performed at each increment, finite element (FE) computations of industrial structures are excessively long. Additionnally, they still do not give a very precise description of the cyclic response, which is required in case of fatigue analysis. 
Phenomenological models consider the material from a macroscopic point of view; they are better suited for structural analysis. On the other hand, they usually involve numerous material parameters (especially in case of anisotropy), the identification of which being possibly tricky and fastidious. This approach has thus not been very applied to SGFR polymers, and works regarding the use of phenomenological models for this kind of material are often limited to random orientation of the short fibres [16-19]. However, Andriyana et al. [20] have proposed a phenomenological viscoelastic-viscoplastic model for the nonlinear response, in which the viscoplastic flow is formulated according to the fibre orientation tensor. This work raises the idea of introducing microstructure information ${ }^{1}$ in a macroscopic model, which combines the advantage of a natural description of the anisotropy with an efficient computation.

We have proposed in a previous work [21] a strategy for a robust identification of a nonlinear constitutive model for SGFR polyamides. The method is based on the uncoupling of the influence of the different parameters, and is validated for complex loading histories. Besides, this approach has been demonstrated as suitable for accurate structural computations [22]. In this work, the same constitutive equations are considered, and are developed in order to explicitely take into account the influence of the microstructure on the mechanical response.

\subsection{Objectives and structure of the paper}

Our goal is the estimation of fatigue life of SGFR polyamides under multiaxial loadings. The adopted approach involves two steps: the computation of the cyclic mechanical response, and the suggestion of a local fatigue criterion. This is the reason why our work is published in two parts. This first part aims at developing the already-published constitutive model for the cyclic behaviour [21], since the mechanical response appears to be both nonlinear and highly anisotropic. Such work is therefore required for an accurate simulation of multiaxial fatigue loadings, and provides input data for any fatigue criterion relying on the knowledge of mechanical values in steady-state. The second step of our approach, i.e. the choice of an appropriate fatigue criterion for SGFR polyamides, is the topic of Part II of this work [1].

The outline of the paper is as follows. Section 2 presents the experimental data which are used in this first part of the work. In Section 3, the constitutive equations for the cyclic behaviour are presented, and we focus on modelling the influence of the microstructure on macroscopic mechanical properties. The proposed constitutive model is validated in Section 4 where numerical predictions are confronted to experimental data for various loading conditions. Section 5 discusses the existence of a steady-state mechanical response and the potential application to the choice of a fatigue criterion. Eventually, final remarks and perspectives are given in Section 6.

\footnotetext{
${ }^{1}$ In this work, the word "microstructure" only refers to the FOD; potential fibre clusters, fibre length distribution or matrix properties heterogeneities (e.g. cristallinity gradient) are neglected and will not be further considered.
} 


\section{Experimental data}

\subsection{Material of the study}

The material of the study is an injection-moulded polyamide 66 reinforced with $35 \mathrm{wt} \%$ of short glass fibres, provided by DuPont de Nemours (DuPont ${ }^{\mathrm{TM}}$ Zytel ${ }^{\circledR} 70 \mathrm{G} 35$ HSLX). In the process of injection, the fibres are oriented according to the viscous flow of the melted material, resulting in an inhomogeneous, anisotropic structure, as represented in Fig. 1. In shell-like structures (the case of most industrial components), the mechanical properties are then likely to vary both across the section thickness and along the spatial location on the shell, in addition to depend on the material direction.

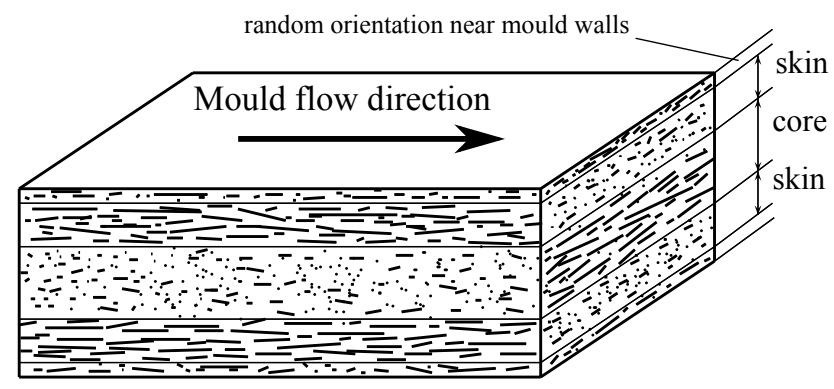

Figure 1: Schematic representation of the skin-core microstructure resulting from the injection moulding process of a fibre reinforced thermoplastic.

The polyamide matrix is also a source of complexity since its mechanical response is highly inelastic, dependent on the strain rate and on environmental conditions such as temperature and water content. The behaviour of the resulting composite thus exhibits both the features of the polyamide and those induced by the distribution of the fibres. Table 1 recalls microstructural and elastic parameters of both matrix and fibre phases of the studied material [21].

Polyamide 66

\begin{tabular}{llll}
\hline Environmental conditions & Young modulus & Poisson coef. \\
$23^{\circ} \mathrm{C}$, DAM & $3064 \mathrm{MPa}$ & 0.38 & \\
$23^{\circ} \mathrm{C}$, RH50 & $1987 \mathrm{MPa}$ & 0.40 & \\
\hline \hline Glass fibres & & & \\
\hline Volume fraction & Young modulus & Poisson coef. & Aspect ratio \\
$19.5 \%$ & $72000 \mathrm{MPa}$ & 0.22 & 25
\end{tabular}

Table 1: Physical and mechanical properties of the studied PA66 GF35 at room temperature, in dry-as-moulded state (DAM), or conditioned with air containing $50 \%$ of relative humidity (RH50). 


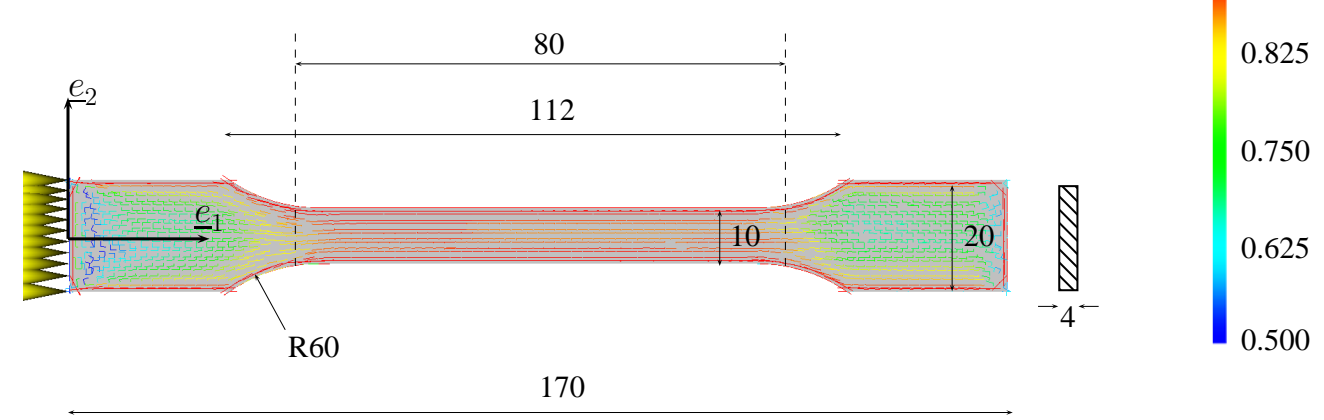

Figure 2: Injection simulation of the ISO527-2-1A tensile specimen with MOLDFLOW, and display of the average fibre orientation over the thickness $\left\langle a_{1}\right\rangle=\int_{0}^{4} a_{1}(z) \mathrm{d} z .4242$ triangular shell elements with 21 Gauss points through the thickness are used for the numerical computation ("Fusion analysis").

\subsection{Experimental conditions and samples}

In this paper (part I), the material is always considered at room temperature $\left(T=23^{\circ} \mathrm{C}\right)$ and in conditioned state (RH50). Two kinds of tensile specimens are studied:

- ISO527-2-1A injection-moulded specimens (see Fig. 2), which have been used for the investigation of the nonlinear cyclic response in a previous work [21];

- IOS527-2-5A specimens milled out of plates, at different angles $\alpha=0,30,45,60,90^{\circ}$ (see Fig. 3). Moreover, when the tensile specimen is parallel to the moulding flow direction $\left(\alpha=0^{\circ}\right)$, it may be milled out of the plate in the central part or near the edge, where short fibres are aligned with more intensity.

A first description of the local FOD of each specimen is the second-order orientation tensor ${ }^{2}$, which is averaged through the thickness and expressed in the global axes as: $\left(\underline{e}_{1}, \underline{e}_{2}, \underline{e}_{3}\right)$ :

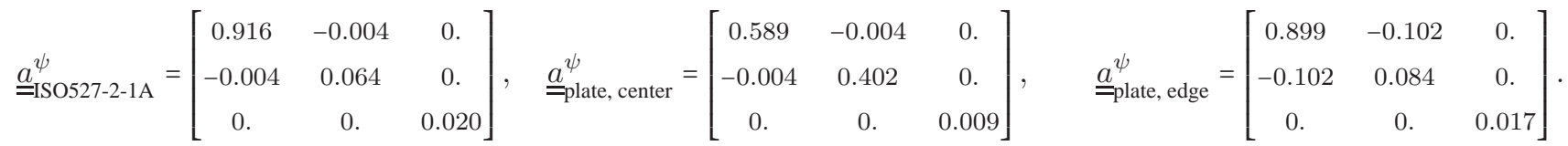

One should notice in Figs. 2 and 3 that the FOD in the gauge length of the tensile specimens is quite homogeneous.

\section{Modelling the cyclic response of the composite}

The mechanical response of SGFR thermoplastics, and especially of the studied PA66 GF35, is highly nonlinear. The designer thus needs a constitutive model in order to describe the local mechanical variables, such as the dissipated

\footnotetext{
${ }^{2}$ See Sec. 3.2.1 for the meaning of notations regarding orientation tensors.
} 


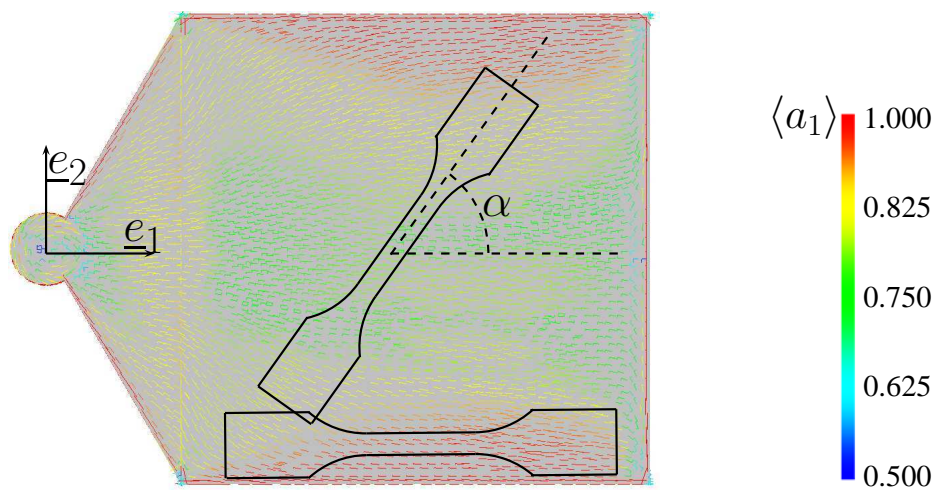

Figure 3: Injection simulation of the plate with MOLDFLOW, and display of the average fibre orientation over the thickness $\left\langle a_{1}\right\rangle=\int_{0}^{2} a_{1}(z) \mathrm{d} z$. The plate dimension is $100 \times 100 \times 2 \mathrm{~mm}^{3}$ whereas the ISO527-2-5A tensile specimen length is $75 \mathrm{~mm}$ (gauge length $20 \mathrm{~mm}$, section $4 \times 2 \mathrm{~mm}^{2}$ ). 5476 triangular shell elements with 21 Gauss points through the thickness are used for the numerical computation ("Fusion analysis").

energy, the cumulated plastic strain, etc. A phenomenological approach for the cyclic behaviour has been proposed by the authors [21].

The ability of the proposed model to describe complex loading histories has been demonstrated on a specific grade of PA66 GF35 in DAM as well as RH50 conditions, at room temperature [21]. Loading rate dependency of the apparent stiffness, long-term creep or relaxation effects have been successfully reproduced at low stress levels thanks to Kelvin-Voigt elements. At higher stress levels, nonlinear viscoplastic mechanisms are activated, along with nonlinear kinematic hardening. At last, a cyclic stiffness softening has been depicted as a function of the viscoplastic dissipated energy. The model has been identified and validated for one specific tensile sample geometry. In the following, the proposed model is extended in order to take into account other microstructures.

\subsection{Constitutive equations}

The constitutive equations developed and physically justified in [21] are briefly recalled. We here emphasize the link between the local fibre orientation and the overall mechanical properties.

The model lies in the framework of the generalized standard materials (GSM) [23, 24]. Five tensorial and one scalar state variables are needed to define the current mechanical state, as well as their associated thermodynamic forces:

- the overall strain $\underline{\underline{\varepsilon}}$, external control variable, associated to the overall stress tensor $\underline{\underline{\sigma}}$;

- the long-term viscoelastic strain $\underline{\underline{\varepsilon}}_{v 1}$, associated to the thermodynamic force for long-term viscoelasticity $\underline{\underline{\mathcal{A}}}_{v 1}$;

- the short-term viscoelastic strain $\underline{\underline{\varepsilon}}_{v 2}$, associated to $\underline{\underline{\mathcal{A}}}_{v 2}$;

- the viscoplastic strain $\underline{\underline{\varepsilon}}_{v p}$, associated to $\underline{\underline{\mathcal{A}}}_{v p}$; 
- the hardening variable $\underline{\underline{\alpha}}$, associated to the center of the pseudo-viscoplastic surface $\underline{\underline{X}}$;

- the cyclic stiffness softening variable, called $\beta$, and associated to $\mathcal{A}_{\beta}$.

The overall strain is decomposed into :

$$
\underline{\underline{\varepsilon}}=\underline{\underline{\varepsilon}}_{e}+\underline{\underline{\varepsilon}}_{v 1}+\underline{\underline{\varepsilon}}_{v 2}+\underline{\underline{\varepsilon}}_{v p}
$$

where $\underline{\underline{\varepsilon}}_{e}$ is the instantaneous elastic strain. The state equations define the thermodynamic forces as functions of the state variables:

$$
\begin{aligned}
\underline{\underline{\sigma}} & =\mathbb{C}^{e}(\beta): \underline{\underline{\varepsilon}}_{e} \\
\underline{\underline{\mathcal{A}}}_{v 1} & =\underline{\underline{\sigma}}-\mathbb{C}^{v_{1}}: \underline{\underline{\varepsilon}}_{v 1} \\
\underline{\underline{\mathcal{A}}}_{v 2} & =\underline{\underline{\sigma}}-\mathbb{C}^{v_{2}}: \underline{\underline{\varepsilon}}_{v 2} \\
\underline{\underline{\mathcal{A}}}_{v p} & =\underline{\underline{\sigma}} \\
\underline{\underline{X}} & =-\frac{2 C}{3} \underline{\underline{\alpha}} \\
\mathcal{A}_{\beta} & =-\frac{1}{2} \underline{\underline{\varepsilon}}_{e}: \frac{\partial \mathbb{C}^{e}}{\partial \beta}: \underline{\underline{\varepsilon}}_{e}
\end{aligned}
$$

where $\mathbb{C}^{e}$ and $\mathbb{C}^{v_{i}}$ stands for the fourth-order elastic tensor, used for the instantaneous elasticity or viscoelasticity. $\mathbb{C}^{e}$ is anisotropic and depends on the microstructure (see Sec. 3.2.2), whereas $\mathbb{C}^{v_{1}}$ and $\mathbb{C}^{v_{2}}$ are assumed to be isotropic [20].

The softening depends on the variable $\beta$ according to the following equations:

$$
\begin{aligned}
\mathbb{C}^{e}(\beta) & =g(\beta) \mathbb{C}^{e}(0) \\
\text { with } g(\beta) & =1-a\left(1-\exp \left(\frac{-\beta}{b}\right)\right)
\end{aligned}
$$

The determination of the initial elastic tensor $\mathbb{C}^{e}(0)$ in the general anisotropic case is discussed in Section 3.2.2.

We define a yield function $f$. Its formulation abides by the GSM framework [16, 24]:

$$
f(\underline{\underline{\sigma}}, \underline{\underline{X}}, \underline{\underline{\alpha}})=\mathcal{J}_{v p}+\frac{3 \gamma}{4 C}\left(\underline{\underline{X}}:\left(\frac{2}{3} \mathbb{P}\right): \underline{\underline{X}}-\frac{4 C^{2}}{9} \underline{\underline{\alpha}}:\left(\frac{2}{3} \mathbb{P}\right): \underline{\underline{\alpha}}\right)
$$

$\mathcal{J}_{v p}$ is the equivalent stress built on the tensor $\underline{\underline{\mathcal{A}}}_{v p}$ centered on $\underline{\underline{X}}$ :

$$
\mathcal{J}_{v p}=\sqrt{\left(\underline{\underline{A}}_{v p}-\underline{\underline{X}}\right): \mathbb{P}:\left(\underline{\underline{A}}_{v p}-\underline{\underline{X}}\right)}
$$

$\mathbb{P}$ is a fourth-order tensor introduced to generalize the von Mises (isotropic) equivalent stress to anisotropic microstructures (see Sec. 3.2.3). The evolution equations describe the state variable rates as functions of the thermodynamic 
forces:

$$
\begin{aligned}
\underline{\underline{\dot{\varepsilon}}}_{v 1} & =\frac{3}{2 \eta_{1}} \underline{\underline{\operatorname{dev}}}\left(\underline{\underline{\mathcal{A}}}_{v 1}\right) \\
\underline{\underline{\dot{\varepsilon}}}_{v 2} & =\frac{3}{2 \eta_{2}} \underline{\underline{\underline{\operatorname{dev}}}}\left(\underline{\underline{\mathcal{A}}}_{v 2}\right) \\
\underline{\underline{\underline{\varepsilon}}}_{v p} & =A\left[\sinh \left(\frac{\mathcal{J}}{H}\right)\right]^{m} \cdot \frac{\mathbb{P}:\left(\underline{\underline{\mathcal{A}}}_{v p}-\underline{\underline{X}}\right)}{\mathcal{J}_{v p}} \equiv \dot{p} \cdot \underline{\underline{n}}_{v p} \\
\underline{\underline{\alpha}} & =-\dot{p}\left(\underline{\underline{n}}_{v p}+\frac{2}{3} \gamma \mathbb{P}: \underline{\underline{\alpha}}\right) \\
\dot{\beta} & =\dot{\underline{\varepsilon}}_{v p}: \mathbb{C}^{e}(\beta): \underline{\underline{\varepsilon}}_{e}-\frac{2 C}{3} \underline{\underline{\dot{\alpha}}}: \underline{\underline{\alpha}}
\end{aligned}
$$

Let us remark that Eq. 13 does not explicitely involve a plastic threshold. The formulation with an hyperbolic sine is similar to the model of Delobelle et al. [25]. According to the GSM framework, the instantaneous dissipated energy density equals the sum of the rates of each state variable multiplied by its associated thermodynamic force:

$$
\mathscr{D}=\underline{\underline{\mathcal{A}}}_{v 1}: \underline{\underline{\dot{\varepsilon}}}_{v 1}+\underline{\underline{\mathcal{A}}}_{v 2}: \underline{\underline{\dot{\varepsilon}}}_{v 2}+\underline{\underline{\mathcal{A}}}_{v p}: \underline{\underline{\dot{\varepsilon}}}_{v p}+\underline{\underline{X}}: \underline{\underline{\dot{\alpha}}}+\mathcal{A}_{\beta} \cdot \dot{\beta}
$$

\subsection{Taking into account the influence of the microstructure}

\subsubsection{Local microstructure and orientation tensors}

The microstructure of a sample made of SGFR thermoplastics is defined by the local fibre orientation distribution $\psi$. As this quantity cannot be computed for a whole structure, injection simulation softwares usually predict the statistical moments of this distribution. These moments are called orientation tensors, according to Advani and Tucker III [26]. $\underline{a}^{\psi}$ and $\mathbb{A}^{\psi}$ denote the second and fourth-order orientation tensor respectively. More specifically, only $\underline{a}^{\psi}$ is computed by injection simulation softwares; $\mathbb{A}^{\psi}$ is extrapolated from $\underline{\underline{a}}^{\psi}$ with a closure approximation.

In this work, we adopte the smooth orthotropic closure equation, suggested by Cintra and Tucker III [27]. If we call $\underline{u}_{i}$ the 3 eigenvectors of $\underline{\underline{a}}^{\psi}$, associated to the eigenvalues $a_{i}$ (with $a_{1} \geqslant a_{2} \geqslant a_{3}$, and $\sum_{i} a_{i}=1$ ), the fourth-order orientation tensor is supposed to be orthotropic in the basis $\left(\underline{u}_{i}\right)_{i=1,2,3}$. Its components are linear combinations of $a_{1}$ and $a_{2}$, detailed in [27].

\subsubsection{Influence of the microstructure on elastic properties}

Overall elastic properties of a SGFR composite with any kind of microstructure (not necessarily random or unidirectional) can be estimated by linear homogenization schemes. For example, the two-step homogenization method has been proposed by Camacho et al. [28] and has been widely used in the literature [29-32].

The first step is the determination of an equivalent unidirectional composite, with for example the Mori-Tanaka scheme. The overall mechanical elastic tensor $\mathbb{C}^{U D}$ is transversely isotropic. $\mathbb{C}^{U D}$ is characterized by five elastic constants $K_{i}$. The second step is an orientation average of these elementary UD composites, according to their orientation. A stiffness averaging is often suggested in the literature, which consists in a Voigt upper bound:

$$
\mathbb{C}^{e}(0)=\int_{\Omega} \mathbb{C}^{\mathrm{UD}}(\underline{p}) \psi(\underline{p}) \mathrm{d} \omega
$$


This integral can be analytically determined if one knows the second and fourth-order orientation tensors:

$$
\mathbb{C}^{e}(0)=K_{1} \mathbb{A}^{\psi}+K_{2}\left(\underline{\underline{a}}^{\psi} \otimes \underline{\underline{1}}+\underline{\underline{1}} \otimes \underline{\underline{a}}^{\psi}\right)+K_{3} \mathbb{D}\left(\underline{\underline{a}}^{\psi}, \underline{\underline{1}}\right)+3 K_{4} \mathbb{J}+2 K_{5} \mathbb{I}
$$

We recall the expression of the five independant constants of a transversely isotropic stiffness tensor, as proposed by Tandon and Weng [33]:

$$
\begin{aligned}
& K_{1}=C_{1111}^{\mathrm{UD}}-2 C_{1122}^{\mathrm{UD}}+C_{2233}^{\mathrm{UD}}-4 C_{1212}^{\mathrm{UD}}+2 C_{2323}^{\mathrm{UD}} \\
& K_{2}=C_{1122}^{\mathrm{UD}}-C_{2233}^{\mathrm{UD}} \\
& K_{3}=C_{1212}^{\mathrm{UD}}-C_{2323}^{\mathrm{UD}} \\
& K_{4}=C_{2233}^{\mathrm{UD}} \\
& K_{5}=C_{2323}^{\mathrm{UD}}
\end{aligned}
$$

In Eq. 18, we use the classical decomposition of the fourth-order unity tensor into spherical and deviatoric projectors $\mathbb{I}=\mathbb{J}+\mathbb{K}$. At last, $\mathbb{D}$ is an operator on second-order tensors defined by its components as $D_{i j k l}\left(\underline{\underline{a}}^{\psi}, \underline{\underline{1}}\right)=a_{i k}^{\psi} \delta_{j l}+$ $a_{i l}^{\psi} \delta_{j k}+a_{j l}^{\psi} \delta_{i k}+a_{j k}^{\psi} \delta_{i l}$.

\subsubsection{Influence of the microstructure on viscoplastic properties}

The local fibre orientation distribution also affects the viscoplastic properties [20]. When loading a composite in the direction where many fibres are aligned, it is obvious that the mechanical response is stiffer, but also less ductile than when loading in a direction where few fibres are aligned. This explains why the viscoplastic equivalent stress $\mathcal{J}_{v p}$ must therefore be built according to an anisotropic tensor $\mathbb{P}$.

According to Hill [34]'s theory, $\mathbb{P}$ is orthotropic in local material axes $\left(\underline{u}_{i}\right)_{i=1,2,3}$. Physically, $\mathbb{P}$ should lead to the isotropic von Mises norm in the case of a random microstructure (isotropic case):

$$
\underline{\underline{a}}^{\psi}=\frac{1}{3} \underline{\underline{ }} \quad \Rightarrow \quad \mathbb{P} \propto \frac{3}{2} \mathbb{K}
$$

In the general case, its components in directions with few aligned fibres must be greater, so that the equivalent viscoplastic stress $\mathcal{J}_{v p}$ is more important, and the viscoplastic mechanisms are more easily activated. Hence, we propose the following expression for $\mathbb{P}$ :

$$
\mathbb{P}=\left[\begin{array}{cccccc}
2 B & D-(B+C) & C-(B+D) & 0 & 0 & 0 \\
D-(B+C) & 2 C & B-(C+D) & 0 & 0 & 0 \\
C-(B+D) & B-(C+D) & 2 D & 0 & 0 & 0 \\
0 & 0 & 0 & 2 E & 0 & 0 \\
0 & 0 & 0 & 0 & 2 E & 0 \\
0 & 0 & 0 & 0 & 0 & 2 E
\end{array}\right]_{\left(\underline{u}_{i}\right)}
$$

with $2 B=\left(1-a_{1}\right)^{k}, 2 C=\left(1-a_{2}\right)^{k}, 2 D=\left(1-a_{3}\right)^{k}$ and $2 E=\left(\frac{2}{3}\right)^{k-1} \cdot k$ is an additional material parameter introduced to describe the sensitivity of the viscoplastic mechanisms on the orientation distribution. We experimentally 
observed that a small variation of the orientation degree around a highly oriented microstructure $\left(a_{1}\right.$ close to 1$)$ has a greater effect on the overall mechanical properties, than a small variation around a poorly orientation microstructure ( $a_{1}$ equals $1 / 3$ for a random distribution). With help of Fig. 4, one can conclude that this observation should lead to values of $k$ between 0 and 1 .

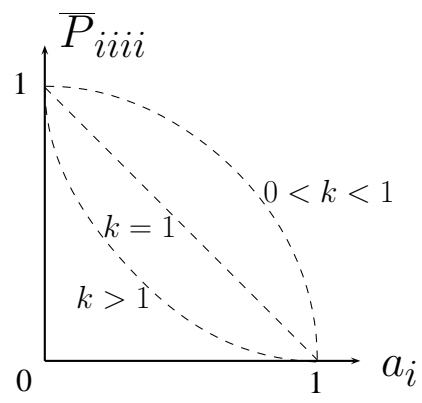

Figure 4: Evolution of the components iiii of tensor $\mathbb{P}$ (expressed in material axes) according to the eigenvalue $a_{i}$ of the second-order orientation tensor.

\section{Numerical results}

\subsection{Monotonic tensile tests}

The proposed model for the nonlinear anisotropic mechanical response has been validated on ISO527-2-5A tensile samples milled out of injected plates (see Fig. 3). The comparison between experimental data and numerical predictions on monotonic tensions at two different loading rate is displayed in Fig. 5.

Let us remark that all material parameters are those identified on injected tensile specimen ISO527-2-1A, as presented in [21]. The parameter $k$ is additionally estimated by a least-square method from experimental results; its value is 0.53 , which is consistent with its physical interpretation (see Sect. 3.2.3).

\subsection{Complex loading histories}

In Fig. 6, the same comparison between experimental data and numerical predictions is made for complex loading cases:

- anhysteretic curves, which are a strain-controlled tensile tests interrupted by relaxation steps of 15 minutes;

- cyclic creep-recovery tests, which are stress-controlled tensile tests with creep and recovery steps of 15 minutes. The imposed stress is increasing at each creep step, whereas the stress during recovery steps is always null.

These tests are convenient to illustrate the highly nonlinear mechanical response and are full validation cases. The compromise between modelling complexity (only one additional parameter, $k$ - previously identified on monotonic 


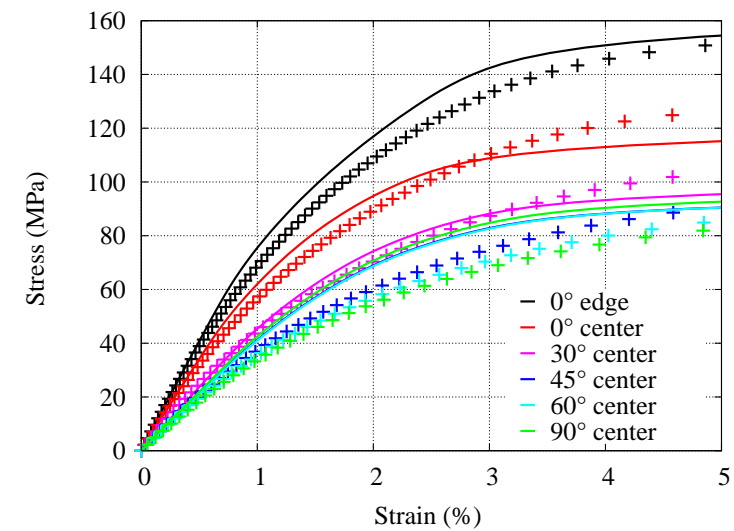

(a) $2.5 \mathrm{MPa} / \mathrm{s}$

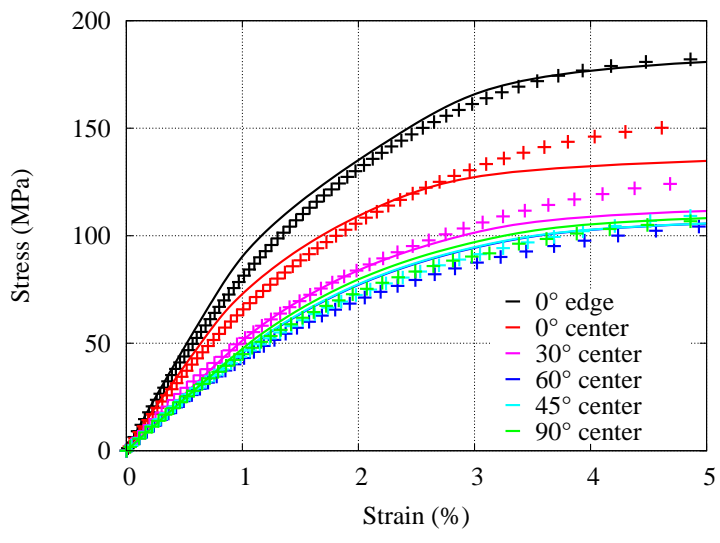

(b) $250 \mathrm{MPa} / \mathrm{s}$

Figure 5: Numerical predictions (continuous lines) versus experimental data (symbols) on specimen milled out of injected plates at different angles, for monotonic tensile tests.

tensions) and the accuracy of the mechanical prediction is fairly good. The proposed constitutive model is thus able to reproduce complex loading histories on various microstructures.

One should notice that we implicitely made the hypothesis of isotropic viscoelasticity in the model, since no influence of the microstructure affects both long- and short-term viscoelastic responses. A possible improvement of the model would consist in either suggesting a phenomenologic expression of the anisotropic viscoelastic moduli $\mathbb{C}^{v_{i}}$ and $\mathbb{H}^{v_{i}}$ (3D anisotropic generalization of scalar parameter $\eta_{i}$ ) or using homogenization schemes developed for linear viscoelastic composites [15]. Such a work could improve the numerical predictions of creep steps (for example) when the fibres are poorly oriented along the mechanical loading direction. However, the identification strategy may be more complex or the computation time may be harshly increased. As the initial stiffness is well predicted at $2.5 \mathrm{MPa} / \mathrm{sas}$ well as $250 \mathrm{MPa} / \mathrm{s}$ for all material orientations (see Fig. 5), the hypothesis of isotropic short-term viscoelasticity is mainly justified. The above-mentioned improvement would thus essentially regard the long-term viscoelastic response. In view of the current extend of our work, we consider that the proposed model stands for a good compromise.

\subsection{Cyclic loading}

Figure 7 displays the application of the proposed constitutive model to cyclic loading. An injection moulded ISO527-2-1A tensile sample is subjected to a sinusoidal stress signal at frequency $f=1 \mathrm{~Hz}$, at amplitude $\sigma_{a}=54$ $\mathrm{MPa}$, and at load ratio $R=0$. The numerical response fits well the experimental data over the first 20 cycles (after which the mechanical evolution is steady - see Sec. 5); the residual strain evolution as well as the hysteretic area are especially fairly predicted.

The constitutive model is thus suitable for the description of cyclic loading in the nonlinear domain (where ir- 


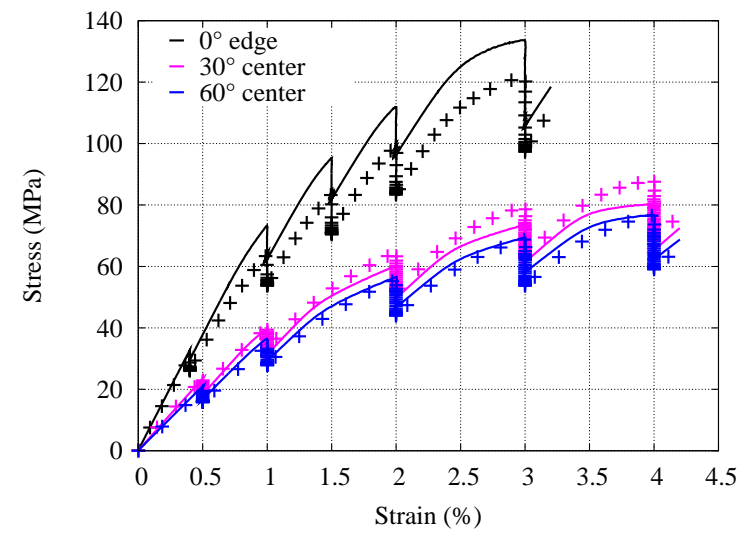

(a) Tension at $0.3 \% / \mathrm{s}$ interrupted by relaxation steps

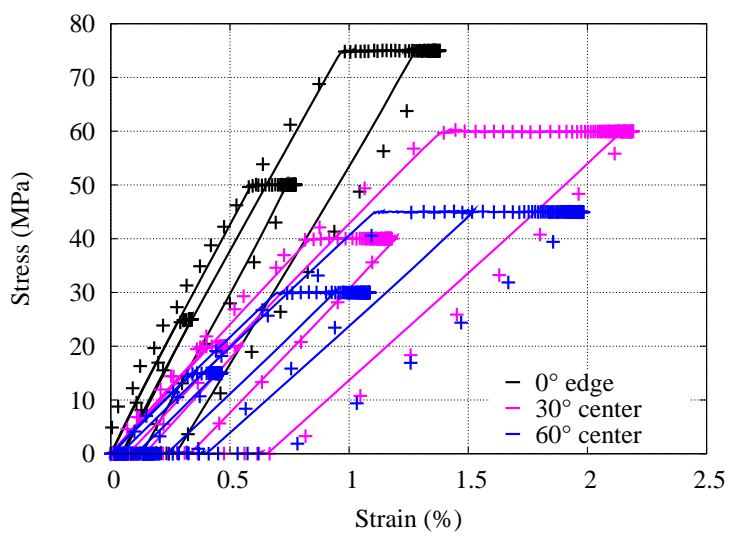

(b) Cyclic creep-recovery test, with increasing stress levels

Figure 6: Numerical predictions (continuous lines) versus experimental data (symbols) on specimen milled out of injected plates at different angles, for complex loading histories.

recoverable strains are activated). Additional experimental data regarding cyclic loadings for different orientation distributions or multiaxial loadings would be yet interesting.

\section{Discussion on the existence of a steady-state mechanical response}

Our approach for fatigue design relies on the uncoupling between the determination of the cyclic mechanical response and the application of a local fatigue criterion. Such an approach is justified since the response of the material reaches a steady-state under fatigue loading. As depicted in Fig. 8, the experimental response may be interpreted as the superposition of a cyclic part $\varepsilon(t)-\bar{\varepsilon}$ and a static evolution of the mean strain $\bar{\varepsilon}$. This result is very similar to the observations made by Bernasconi et al. [35]. Besides, Klimkeit et al. [9], as well as De Monte et al. [36], showed that the static evolution depends on the load ratio and may be attributed to creep-fatigue coupling.

Figure 9a displays the evolution of the hysteretic area $\Delta W_{\text {tot }}=\int_{\text {cycle }} \sigma \mathrm{d} \varepsilon$ according to the number of cycles $N$. It is shown that this mechanical value reaches a stabilized value after a few number of cycles, estimated at 20 in our case. We consider that the cyclic part of the mechanical response has then reached a steady-state; in that case, the hysteretic area equals the dissipated energy density per cycle, $\Delta W_{\text {diss }}=\int_{\text {cycle }} \mathscr{D} \mathrm{d} t$. In the same manner, the evolution of the residual strain, which is an indicator of cumulated viscoplastic strain, reaches an asymptotic linear evolution after 20 cycles: the residual strain increment is then nearly constant.

The numerical simulation of load-controlled fatigue tests over 20 cycles gives access to some mechanical values which are constant during fatigue life (e.g. stress components or stress invariants, provided that stress redistributions are negligible), which reach a steady-state (e.g. dissipated energy density per cycle, plastic strain increment per cycle), or which may continue to evolve (e.g. maximum strain, cumulated plastic strain). With help of Fig. 7, it is clear 


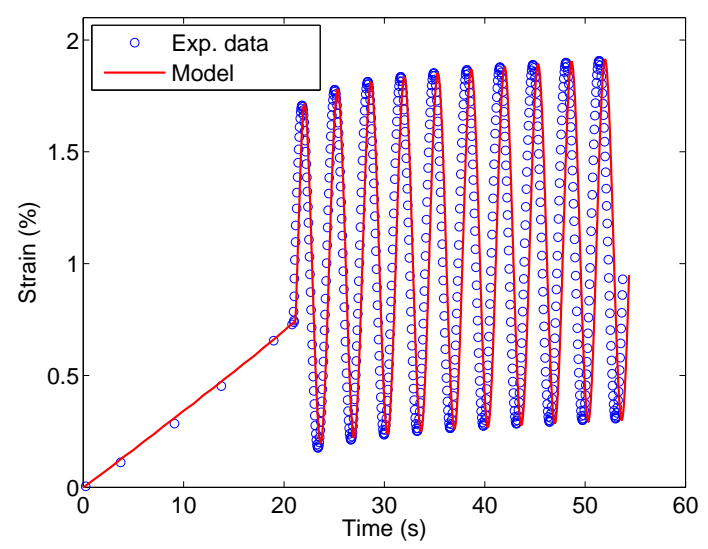

(a) Strain evolution during the first 10 cycles

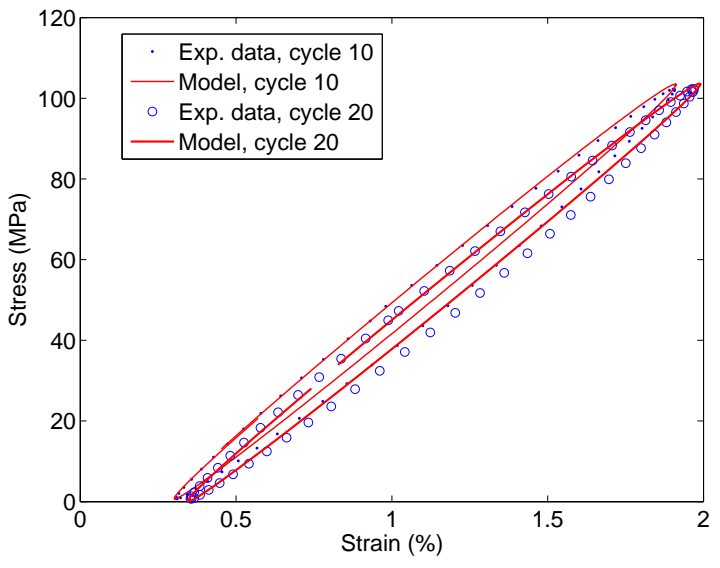

(b) Stress-strain response at cycles 10 and 20

Figure 7: Numerical predictions of the proposed constitutive model versus experimental data on injection moulded ISO527-2-1A tensile specimen under sinusoidal stress-controlled loading.

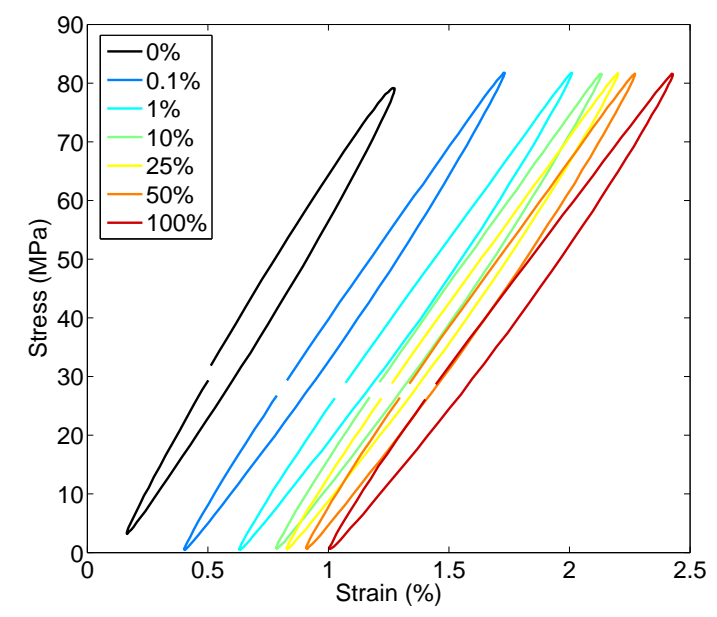

(a) Stress-strain response during fatigue test

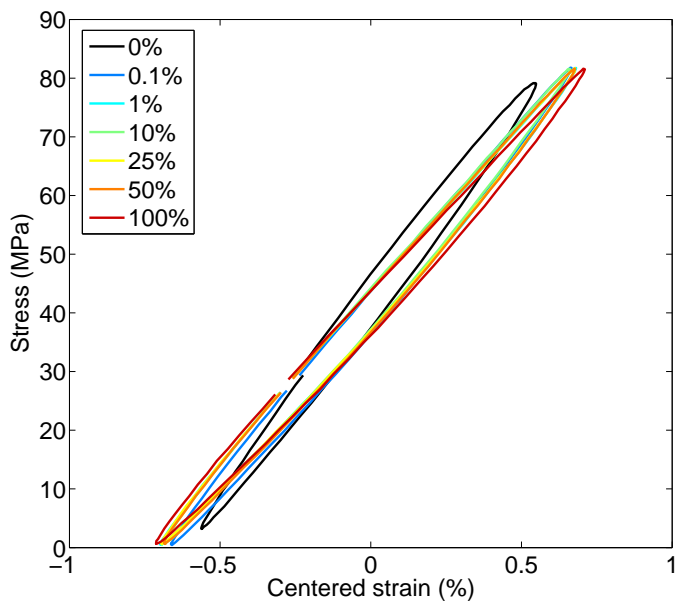

(b) Stress versus centered strain

Figure 8: Evolution of the mechanical response during fatigue life (expressed in percentage of lifetime) of an injection moulded ISO527-2-1A sample under stress-controlled sinusoidal loading. The centered strain is defined as $\varepsilon(t)-\bar{\varepsilon}$, where $\bar{\varepsilon}$ is the mean strain over the current cycle. 


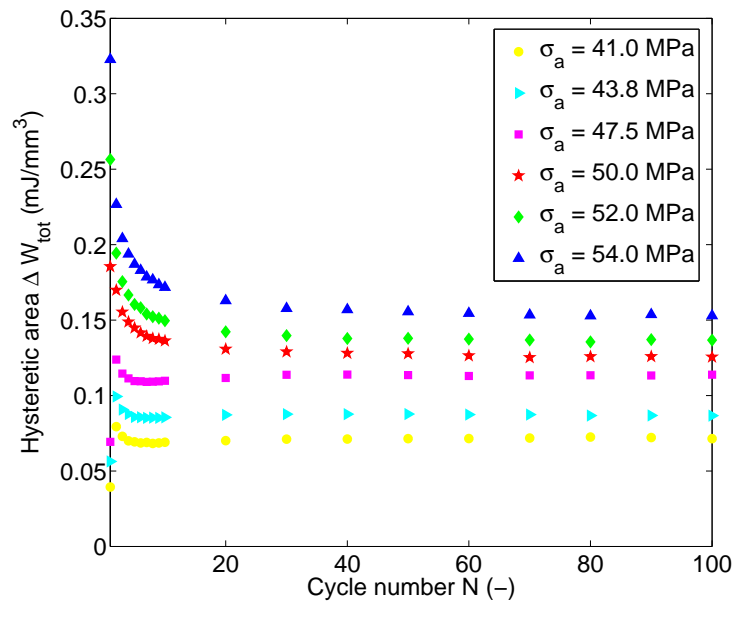

(a) Hysteretic area $\Delta W_{\text {tot }}$

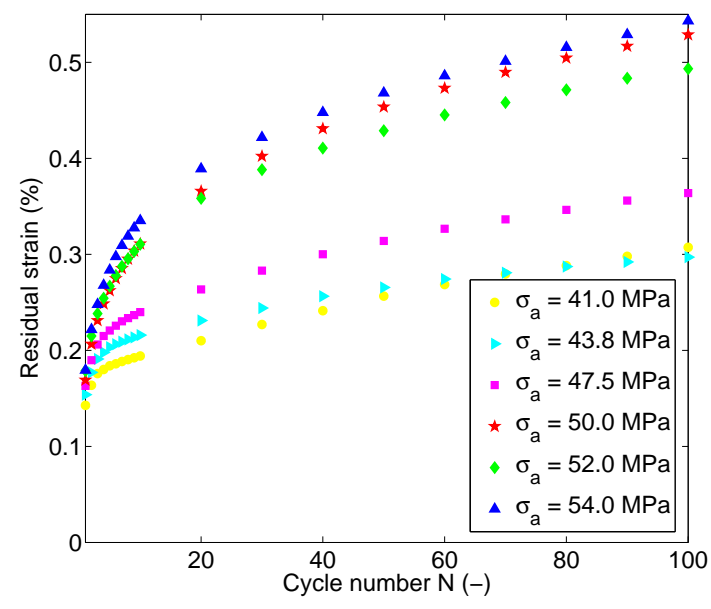

(b) Residual strain $\varepsilon(\sigma=0)$

Figure 9: Evolution of the hysteretic area and the residual strain during tensile-tensile cyclic loadings on injection moulded ISO527-2-1A samples at various stress amplitude $(f=1 \mathrm{~Hz}, R=0)$.

that the numerical computation over 20 cycles gives fairly confident values for the determination of the mechanical characterizing the steady-state: dissipated energy density, visplastic strain increment, stress or strain amplitudes, etc. At last, one must be aware that the uncoupling between the simulation of the cyclic response and the application of a fatigue criterion is fully justified regarding constant or stabilized mechanical values, but should be considered with caution regarding still-evolving ones. This point will be deepened in Part II of this work [1].

\section{Conclusions}

This paper deals with the mechanical behaviour of short glass fibre reinforced polyamides under static and cyclic tensile load. Constitutive equations proposed and validated in a previous work for one specific fibre orientation distribution are generalized in order to take into account the influence of the microstructure on the mechanical properties. A classical two-step homogenization scheme is used for the elastic tensor, whereas an original form of the Hill-like equivalent viscoplastic stress is suggested, relying on the second-order orientation tensor. The proposed model is validated for different load histories and different fibre orientations; only one additional material parameter is identified to capture the anisotropy of the mechanical response in the nonlinear domain.

The proposed model is applied to stress-controlled cyclic tensile tests. It is shown that the numerical prediction, calibrated on static and stepped static tensile histories, displays a good agreement with the experimental data. The existence of a steady-state is discussed, and it is shown that 20 cycles are sufficient to observe a stabilization of some macroscopic quantities, such as the dissipated energy density or the viscoplastic strain increment. However, cyclic 
loadings on tensile samples with different fibre orientations would be interesting in order to generalize these first results to various microstructure.

Part II of this work will regard the application of the proposed constitutive model to multiaxial fatigue tests published in the literature about SGFR polyamide [2,3]. The goal is the choice of a criterion to estimate their fatigue life under multiaxial fatigue loading which are representative of actual loadings encountered during the service life on vehicules.

\section{Acknowledgement}

This work was supported by PSA Peugeot-Citroën and has received the financial support of the French Minister for Research (ANRT). The authors gratefully acknowledge DuPont de Nemours for providing material data and specimens.

\section{References}

[1] Launay, A., Maitournam, M., Marco, Y., Raoult, I.. Multiaxial fatigue models for short glass fibre reinforced polyamide. Part II: Fatigue life estimation. International Journal of Fatigue (submitted for publication) 2012;

[2] De Monte, M., Moosbugger, E., Jasckek, K., Quaresimin, M.. Multiaxial fatigue of a short fibre reinforced polyamide 6.6 - Fatigue and fracture behaviour. International Journal of Fatigue 2010;32:17-28.

[3] Klimkeit, B.. Etude expérimentale et modélisation du comportement en fatigue multiaxiale d'un polymère renforcé pour application automobile. Ph.D. Thesis; École Nationale Supérieure de Mécanique et d'Aérotechnique; 2009.

[4] Meraghni, F., Desrumaux, F., Benzeggagh, M.. Implementation of a constitutive micromechanical model for damage analysis in glass mat reinforced composite structures. Composites Science and Technology 2002;62(16):2087-2097.

[5] Nouri, H., Meraghni, F., Lory, P.. Fatigue damage model for injection-molded short glass fibre reinforced thermoplastics. International Journal of Fatigue 2009;31(5):934-942.

[6] Charkaluk, E., Constantinescu, A.. An energetic approach in thermomechanical fatigue for silicon molybdenum cast iron. Materials at High Temperatures 2000;17(3):373-380.

[7] Constantinescu, A., Charkaluk, E., Lederer, G., Verger, L.. A computational approach to thermomechanical fatigue. International Journal of Fatigue 2004;26(8):805-818.

[8] Raoult, I.. Structures élastomères sous chargement cyclique : Comportement - fatigue - durée de vie. Ph.D. Thesis; École Polytechnique; Palaiseau; 2005.

[9] Klimkeit, B., Castagnet, S., Nadot, Y., El Habib, A., Benoit, G., Bergamo, S., et al. Fatigue damage mechanisms in short fiber reinforced PBT+PET GF30. Mechanical Science and Engineering A 2011;528:1577-88.

[10] Mourglia Seignobos, E.. Compréhension des mécanismes physiques de fatigue dans le polyamide vierge et renforcé de fibres de verre. Ph.D. Thesis; INSA de Lyon; 2009.

[11] Noda, K., Takahara, A., Kajiyama, T.. Fatigue failure mechanisms of short glass-fiber reinforced nylon 66 based on nonlinear dynamic viscoelastic measurement. Polymer 2001;42(13):5803-5811.

[12] Doghri, I., Ouaar, A.. Homogenization of two-phase elasto-plastic composite materials and structures. Study of tangent operators, cyclic plasticity and numerical algorithms. International Journal of Solids and Structures 2003;40(7):1681-1712.

[13] Doghri, I., Friebel, C.. Effective elasto-plastic properties of inclusion-reinforced composites. Study of shape, orientation and cyclic response. Mechanics of Materials 2005;37(1):45-68. 
[14] Doghri, I., Tinel, L.. Micromechanical modeling and computation of elasto-plastic materials reinforced with distributed-orientation fibers. International Journal of Plasticity 2005;21(10):1919-1940.

[15] Friebel, C., Doghri, I., Legat, V.. General mean-field homogenization schemes for viscoelastic composites containing multiple phases of coated inclusions. International Journal of Solids and Structures 2006;43(9):2513-2541.

[16] Chaboche, J.. Thermodynamic formulation of constitutive equations and application to the viscoplasticity and viscoelasticity of metals and polymers. International Journal of Solids and Structures 1997;34(18):2239-2254.

[17] Drozdov, A., Al-Mulla, A., Gupta, R.. The viscoelastic and viscoplastic behavior of polymer composites: polycarbonate reinforced with short fibers. Computational Materials Science 2003;28:16-30.

[18] Drozdov, A., Al-Mulla, A., Gupta, R.. Finite viscoplasticity of polycarbonate reinforced with short glass fibers. Mechanics of Materials 2005;37(4):473-491.

[19] Rémond, Y.. Constitutive modelling of viscoelastic unloading of short glass fibre-reinforced polyethylene. Composites Science and Technology 2005;65(3-4):421-428.

[20] Andriyana, A., Billon, N., Silva, L.. Mechanical response of a short fiber reinforced thermoplastic: Experimental investigation and continuum mechanical modeling. European Journal of Mechanics-A/Solids 2010;29(6):1065-1077.

[21] Launay, A., Maitournam, M., Marco, Y., Raoult, I., Szmytka, F.. Cyclic behavior of short glass fiber reinforced polyamide: experimental study and constitutive equations. International Journal of Plasticity 2011;27:1267-93.

[22] Launay, A., Marco, Y., Maitournam, M., Raoult, I.. Constitutive behavior of injection molded short glass fiber reinforced thermoplastics: a phenomenological approach. Procedia Engineering 2011;10:2003-8.

[23] Halphen, B., Nguyen, Q.S.. Sur les matériaux standards généralisés. Journal de Mécanique 1975;14(1):39-63.

[24] Germain, P., Suquet, P., Nguyen, Q.. Continuum thermodynamics. Journal of Applied Mechanics 1983;50(4):1010-1020.

[25] Delobelle, P., Robinet, P., Geyer, P., Bouffioux, P.. A model to describe the anisotropic viscoplastic behaviour of Zircaloy-4 tubes. Journal of Nuclear Materials 1996;238(2-3):135-162.

[26] Advani, S., Tucker III, C.. The Use of Tensors to Describe and Predict Fiber Orientation in Short Fiber Composites. Journal of Rheology $1987 ; 31: 751$.

[27] Cintra, J., Tucker III, C.. Orthotropic closure approximations for flow-induced fiber orientation. Journal of Rheology 1995;39(6):1095-1122.

[28] Camacho, C., Tucker III, C., Yalvac, S., McGee, R.. Stiffness and thermal expansion predictions for hybrid short fiber composites. Polymer Composites 1990;11(4):229-39.

[29] Lielens, G., Pirotte, P., Couniot, A., Dupret, F., Keunings, R.. Prediction of thermo-mechanical properties for compression moulded composites. Composites Part A 1998;29(1-2):63-70.

[30] Mlekusch, B.. Thermoelastic properties of short-fibre-reinforced thermoplastics. Composites Science and Technology 1999;59:911-923.

[31] Pierard, O., Friebel, C., Doghri, I.. Mean Field homogenization of multi-phase thermo-elastic composites: a general framework and its validation. Composites Science and Technology 2004;64(10-11):1587-1603.

[32] Dray, D., Gilormini, P., Régnier, G.. Comparison of several closure approximations for evaluating the thermoelastic properties of an injection molded short-fiber composite. Composites Science and Technology 2007;67(7-8):1601-1610.

[33] Tandon, G., Weng, G.. The effect of aspect ratio of inclusions on the elastic properties of unidirectionally aligned composites. Polymer Composites 1984;5(4):327-333.

[34] Hill, R.. A theory of the yielding and plastic flow of anisotropic metals. Proceedings of the Royal Society of London Series A, Mathematical and Physical Sciences 1948;193(1033):281-297.

[35] Bernasconi, A., Davoli, P., Basile, A., Filippi, A.. Effect of fibre orientation on the fatigue behaviour of a short glass fibre reinforced polyamide-6. International Journal of Fatigue 2007;29(2):199-208.

[36] De Monte, M., Moosbugger, E., Quaresimin, M.. Influence of temperature and thickness on the off-axis behaviour of short glass fibre reinforced polyamide 6.6 - cyclic loading. Composites: Part A 2010;41:1368-79. 
DuPont ${ }^{\mathrm{TM}}$, and all products denoted with ${ }^{\circledR}$ or ${ }^{\mathrm{TM}}$ are registred trademarks of E. I. du Pont de Nemours and Company or its affiliates. 